\title{
First implantation of the CE-marked transcatheter Sapien pulmonic valve in Europe
}

\author{
Peter Ewert • Eric Horlick · Felix Berger
}

Received: 24 June 2010/Accepted: 19 August 2010/Published online: 18 September 2010

(C) The Author(s) 2010. This article is published with open access at Springerlink.com

\section{Sirs:}

Transcatheter pulmonary valve implantation was first performed in 2000 by Philip Bonhoeffer [1]. As a generally available product (Melody Valve, Medtronic, Minneapolis, MN, USA), the valve was introduced in clinical practise in summer 2007. Since then about 1,500 valves have been implanted worldwide. One of the drawbacks of the Melody valve, however, is its maximal diameter of $22 \mathrm{~mm}$. Parallel to this development, the Edwards SAPIEN aortic valve was evaluated to be implanted into pulmonary position in a small series of patients [2]. Since May 2010 this valve is certified and commercially available in sizes of 23 and $26 \mathrm{~mm}$ diameter as an implant for the pulmonary artery (Edwards Sapien Pulmonic THV, Edwards Lifesciences, Irvine, CA, USA) [3]. We report on the first implantation of this CE-marked valve in Europe.

A 72-year-old woman received a Ross-procedure in 2007 due to a severe combined aortic valve dysfunction. In pulmonary position a decellularized porcine valve (Matrix $\mathrm{P}$, AutoTissue GmbH, Berlin, Germany) was implanted in another institution. Three years after the index operation, this biological valve degenerated and there was severe pulmonary insufficiency with a regurgitation fraction of $60 \%$ and marked right ventricular dilatation measured by MRI. The patient had noted a decline in exercise tolerance for 6 months with NYHA-class III symptoms. She suffered

\footnotetext{
P. Ewert $(\bowtie) \cdot$ F. Berger

Department of Congenital Heart Diseases,

German Heart Institute, Augustenburger Platz 1,

13353 Berlin, Germany

e-mail: ewert@dhzb.de

E. Horlick

Toronto General Hospital, Toronto, Canada
}

from chronic atrial fibrillation and had a left ventricular ejection fraction of $45 \%$ with excellent autograft function.

In a diagnostic catheterization, coronary atherosclerosis was excluded and the pulmonary regurgitation confirmed. Right ventricular systolic pressure was $43 \mathrm{mmHg}$ with a mild gradient at the distal anastomosis of the bioprosthesis to the pulmonary bifurcation of $10 \mathrm{mmHg}$. Angiography revealed large diameters of $22-26 \mathrm{~mm}$ at the level of the valve. Only at the distal anastomosis, a discrete reduction to $19 \mathrm{~mm}$ diameter could be found (Fig. 1a, b). Because no calcification of the valve was present, a sizing manoeuvre was performed with a compliant balloon (AGA Medical, Golden Valley, MN, USA). A minimal diameter of $22 \mathrm{~mm}$ could be found. Simultaneously, a coronary injection ruled out any coronary artery near the implantation site (Fig. 1b). To create a rigid landing zone for the transcatheter valve, the anastomosis was pre-stented: A $48 \mathrm{~mm}$ XXL Andrastent (Andramed $\mathrm{GmbH}$, Reutlingen, Germany) was implanted on a 24 mm BiB-Balloon (NuMED Inc., Hopkinton, NY, USA) and flared at its distal end with a $16 \mathrm{~mm}$ Z-Med-Balloon (NuMED Inc., Hopkinton, NY, USA) inflated simultaneously with the BiB. Then, two $45 \mathrm{~mm}$ CP-Stents (one bare metal, one covered, NuMED Inc., Hopkinton, NY, USA) were subsequently implanted with the same $24 \mathrm{~mm}$ balloon into the target region (Fig. 1c). A $26 \mathrm{~mm}$ transcatheter pulmonary valve was implanted (Edwards Sapien Pulmonic THV, Edwards Lifesciences, Irvine, CA, USA) through a $24 \mathrm{~F}$ sheath (outer diameter $28 \mathrm{~F}$ ) without any problem (Fig. 1d). The valve was angiographically confirmed to be symmetrically configured and competent without any residual pressure gradient. The right ventricular systolic pressure at the end of the procedure was $30 / 0-5 \mathrm{mmHg}$. Fluoroscopy time was $17.2 \mathrm{~min}$.

The introduction of transcatheter pulmonary valves into clinical practice has broadened the interventional 

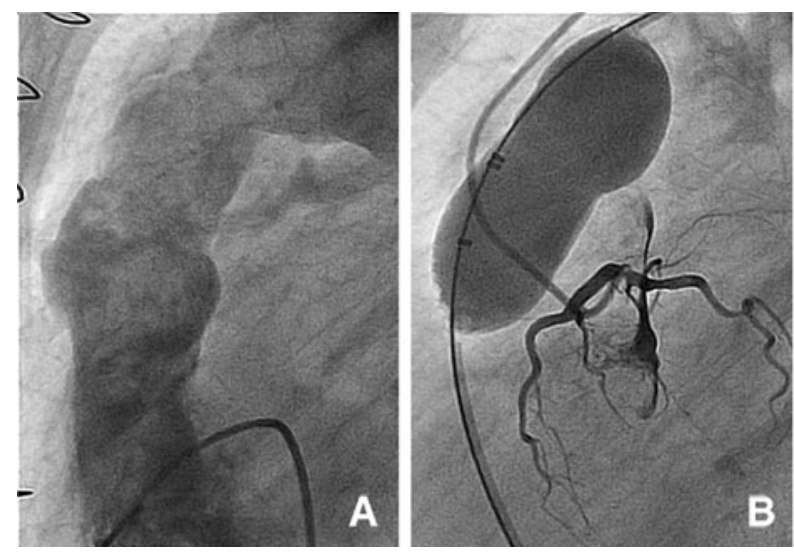

B
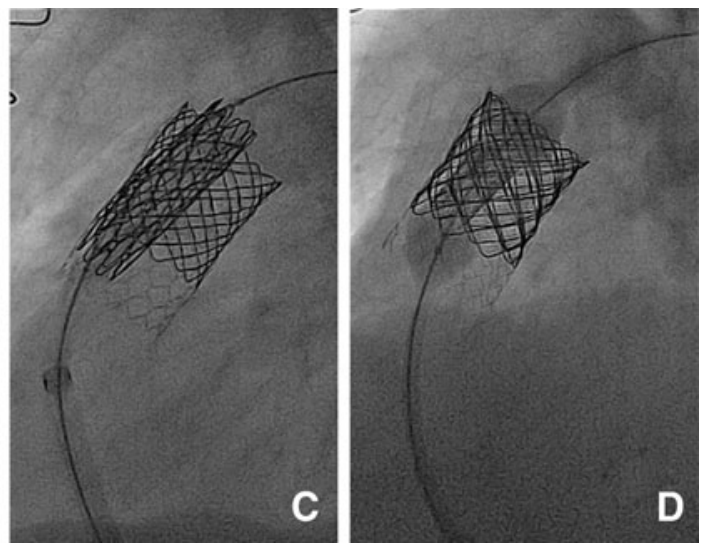

Fig. 1 a Right ventricular angiography: large outflow tract, no valve leaflets, mild narrowing at distal anastomosis of a Matrix $P$ valve. b Balloon sizing of the landing zone: simultaneous angiography of the left coronary artery. The balloon does not impinge on the coronary artery. The mild indentation in the balloon measures $20 \mathrm{~mm}$ in

possibilities in congenital heart diseases and in patients after the Ross-procedure [4, 5]. Both commercially available valves are mounted on balloon expandable stents. Thus, the need of a rigid target zone to safely anchor the valve-carrying stent is mandatory in order to prevent valve dislocation. The Melody valve from Medtronic is an original bovine jugular vein prepared in toto for implantation in man. The valve functions in low pressure conditions and is extremely forgiving with regard to smaller diameters and asymmetry, i.e. the same valve works perfectly well in a range from at least $14 \mathrm{~mm}$ up to $22 \mathrm{~mm}$ diameter as well as in oval configurations. The Sapien pulmonic valve from Edwards Lifesciences is a bovine pericardial valve which comes in two different sizes of 23 and $26 \mathrm{~mm}$ diameter. It works best at the nominal size and tolerates only minor deviations in size and symmetry. Smaller deployment sizes have not yet been tested. In this sense, these products could be used to complement each other. If smaller valve diameters are appropriate, the Melody valve could be used up to $22 \mathrm{~mm}$ diameter (introducer size $22 \mathrm{~F}$ outer diameter), for larger diameters up to $26 \mathrm{~mm}$ the SAPIEN pulmonic valve (introducer size up to $28 \mathrm{~F}$ outer diameter) is available (Fig. 2).

In contrast to the transcatheter implantation of an aortic valve, it is noteworthy that transcatheter pulmonary valves need a landing zone usually prepared by pre-stenting. Thus, the transcatheter implantation of a valve in the pulmonary position is a complex procedure. Often, the most challenging part is not the implantation of the valve itself, but the preparation of the landing zone. The meticulous exclusion of any possible coronary impingement by the implantation of the stents, and the awareness of possible conduit ruptures are possibly the two major factors to have been considered during the procedure. A variety of large diameter. c Two large stents are already in place, a third one is about to be implanted to prepare a rigid landing zone for the valve. d Implantation of the SAPIEN pulmonic valve in the pre-stented pulmonary artery

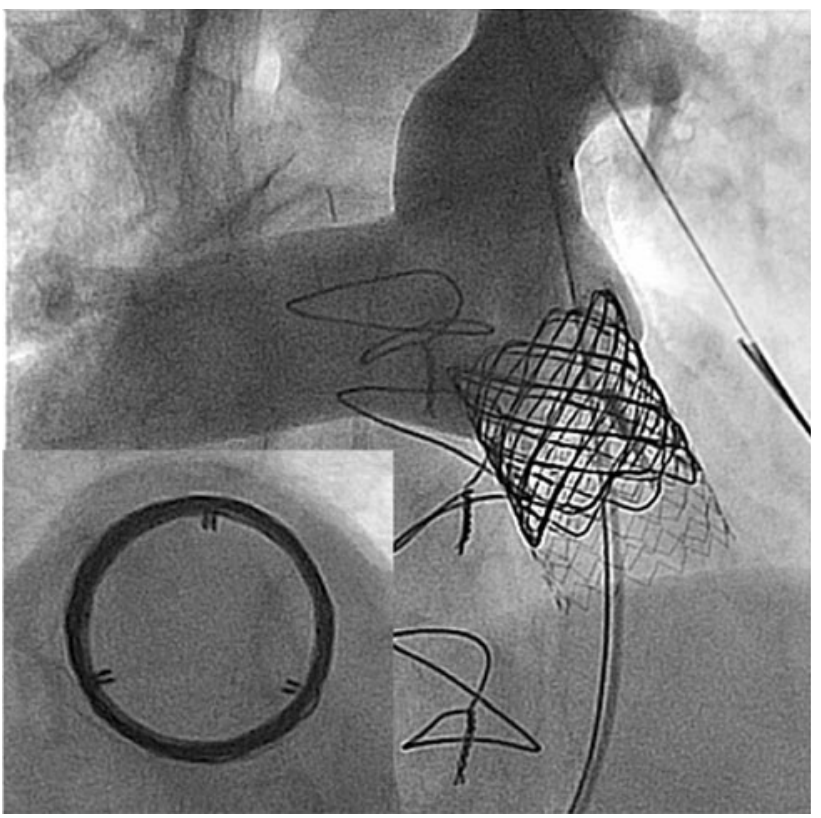

Fig. 2 Final angiography after implantation of three large stents and a $26 \mathrm{~mm}$ SAPIEN pulmonic valve. There is no residual gradient and no pulmonary regurgitation. The valve is expanded completely and symmetrically (small picture in lower left corner)

balloon expandable stents (bare metal and covered), large balloons, sheaths and bail out equipment as well as experience in the use of this equipment are prerequisites to perform a transcatheter pulmonary valve implantation successfully and safely. Due to the relatively small number of cases, we would recommend to perform the procedure only in larger centres with at least 15 transcatheter pulmonary valve implantations per year. 
Open Access This article is distributed under the terms of the Creative Commons Attribution Noncommercial License which permits any noncommercial use, distribution, and reproduction in any medium, provided the original author(s) and source are credited.

\section{References}

1. Bonhoeffer P, Boudjemline Y, Saliba Z, Merckx J, Aggoun Y, Bonnet D, Acar P, Le Bidois J, Sidi D, Kachaner J (2000) Percutaneous replacement of pulmonary valve in a right-ventricle to pulmonary-artery prosthetic conduit with valve dysfunction. Lancet 356(9239):1403-1405
2. Boone RH, Webb JG, Horlick E, Benson L, Cao QL, Nadeem N, Kiess M, Hijazi ZM (2010) Transcatheter pulmonary valve implantation using the Edwards Sapien transcatheter heart valve. Catheter Cardiovasc Interv 75(2):286-294

3. http://wwwmarketwirecom/press-release/Edwards-Lifesciencesgibt-Erhalt-der-CE-Kennzeichnung-fur-seine-kathetergesteuertepulmonale-NYSE-EW-1267037htm

4. Lurz P, Bonhoeffer P, Taylor AM (2009) Percutaneous pulmonary valve implantation: an update. Expert Rev Cardiovasc Ther 7(7):823-833

5. Ewert P, Eicken A, Peters B, Fratz S, Kühne T, Hager A, Ovroutsky S, Vogt M, Berger F, Hess J (2009) Percutaneous pulmonary valve replacement: two-center experience in the first 60 patients (German). Clin Res Cardiol 98(9):592-593 